\title{
Schwarzschild-like black holes: Light-like trajectories and massless scalar absorption
}

\author{
Renan B. Magalhães ${ }^{1, \mathrm{a}}$, Luiz C. S. Leite ${ }^{1,2, \mathrm{~b}} \underset{\mathbb{C}}{ }$, Luís C. B. Crispino ${ }^{1, \mathrm{c}}$ \\ ${ }^{1}$ Programa de Pós-Graduação em Física, Universidade Federal do Pará, Belém, Pará 66075-110, Brazil \\ ${ }^{2}$ Campus Altamira, Instituto Federal do Pará, Altamira, Pará 68377-630, Brazil
}

Received: 3 March 2020 / Accepted: 3 April 2020 / Published online: 8 May 2020

(C) The Author(s) 2020

\begin{abstract}
Black holes are among the most intriguing objects in nature. They are believed to be fully described by General Relativity (GR), and the astrophysical black holes are expected to belong to the Kerr family, obeying the nohair theorems. Alternative theories of gravity or parameterized deviations of GR allow black hole solutions, which have additional parameters other than mass and angular momentum. We analyze a Schwarzschild-like metric, proposed by Johannsen and Psaltis, characterized by its mass and a deformation parameter. We compute the absorption cross section of massless scalar waves for different values of this deformation parameter and compare it with the corresponding scalar absorption cross section of the Schwarzschild black hole. We also present analytical approximations for the absorption cross section in the high-frequency regime. We check the consistence of our results comparing the numerical and analytical approaches, finding excellent agreement.
\end{abstract}

\section{Introduction}

The no-hair theorems establish a paradigm in black hole (BH) physics: BHs belong to the Kerr family [1], so that the astrophysical BH candidates are fully described by two parameters - their mass and total angular momentum [2]. Although this no-hair paradigm has not been refuted by experimental tests of General Relativity (GR) [3-8], the strong field regime of GR is being put to test [9-15], and several works propose deviations to standard GR solutions, by including parameters beyond mass and total angular momentum.

Generalizations of GR solutions have been proposed over the years. Among the approaches are the bumpy BH $[16,17]$ and the modified bumpy BH formalism [18], each of them

\footnotetext{
a e-mail: rbmagalhaes22@hotmail.com

b e-mail: luizcsleite@ufpa.br (corresponding author)

c e-mail: crispino@ufpa.br
}

with advantages and limitations. To surpass the pathologies of bumpy solutions, in 2011 Johannsen and Psaltis proposed, without the constraint of satisfying the Einstein's equations, a Schwarzschild-like spacetime with an additional parameter [19]. They applied the Newman-Janis algorithm and obtained a rotating version of their static solution. Since then, many works analyzed the Johannsen-Psaltis (JP) spacetime, e.g. including charge in the $\mathrm{BH}$ spacetime [20], and computing of the photon orbits in this geometry [21]. Generalizations of JP metric [22], and new parametrized solutions $[23,24]$ have also been proposed.

The decade of 1970 brought a powerful method to analyze the behavior of fields in $\mathrm{BH}$ spacetimes, allowing the study of the absorption and scattering of fields by BHs [2527]. The first works investigated the field equations analytically and the range of validity of the solutions were restrict to some limiting (low- or high-frequency) regimes. With numerical methods the absorption and scattering problems became treatable in the whole frequency range. This numerical technique has been extensively revisited along the years [28-34].

We study a massless scalar field propagating in the vicinity of a non-spinning JP spacetime. We consider a plane wave impinging from infinity and discuss how the JP deformation parameter influences the absorption by the $\mathrm{BH}$. In the eikonal limit, we use the geodesic equation to obtain high-frequency approximations, comparing them to our numerical results.

The remaining of this paper is organized as follows. In Sect. 2 we present the static Johannsen-Psaltis BH (JPBH) and point out some properties of the corresponding spacetime. In Sect. 3 we exhibit the photon orbit equation and solve it to determine the classical absorption cross section, which we use to check the consistency of the numerical results in the high-frequency limit. In Sect. 4 we investigate the massless scalar field, obtaining the radial and angular equations. In Sect. 5 we compute numerically the partial and total absorption cross sections of the massless scalar field for the JPBH. 
We conclude with our final remarks in Sect. 6. We make use of natural units $G=c=\hbar=1$, and signature $(+---)$.

\section{Non-spinning Johannsen-Psaltis BH}

In order to investigate a new class of Schwarzschild-like objects, the JP line element [19], namely

$d s^{2}=[1+h(r)]\left(f(r) d t^{2}-\frac{1}{f(r)} d r^{2}\right)-r^{2} d \Omega^{2}$,

has been introduced, where $f(r) \equiv 1-2 M / r$ and $d \Omega^{2}=$ $d \theta^{2}+\sin ^{2} \theta d \phi^{2}$ is the line element of an unit sphere. The usual Schwarzschild line element is recovered for a vanishing deformation function $h(r)$. Following Ref. [19], we chose $h(r)$ to be a power series of $M / r$, namely

$h(r)=\sum_{k=0}^{\infty} \varepsilon_{k}\left(\frac{M}{r}\right)^{k}$.

The asymptotic flatness of the spacetime, together with the experimental data of Lunar Laser Ranging [35] and Cassini experiments [36] imply in constrains to $h(r)$, reducing it to

$h_{J P}(r)=\varepsilon \frac{M^{3}}{r^{3}}$,

with $\varepsilon_{3} \equiv \varepsilon$. Hence, the non-spinning JP line element (1) takes the form

$d s^{2}=\left[1+\varepsilon\left(\frac{M}{r}\right)^{3}\right]\left[f(r) d t^{2}-\frac{1}{f(r)} d r^{2}\right]-r^{2} d \Omega^{2}$.

The spacetime described by the line element (4) is asymptotically flat, with spherical symmetry, and with a timelike Killing vector field associated to it. The event horizon location is at $r_{h}=2 M$, as in the case of Schwarzschild spacetime. It is important to emphasize that the metric associated to the line element (4) is not required to be a solution of Einstein's equations [19], and that a gravity theory which field equations have the line element (4) as a solution is still unknown.

We can look for the singularities of the non-spinning metrics associated to the line element (4) obtaining the Kretschmann scalar $K=R_{\mu \nu \sigma \rho} R^{\mu \nu \sigma \rho}$, namely

$$
\begin{aligned}
K= & \frac{1}{\left(\frac{M^{3} \varepsilon}{r^{3}}+1\right)^{6}}\left(\frac{48 M^{2}}{r^{6}}-\frac{80 M^{4} \varepsilon}{r^{8}}+\frac{432 M^{5} \varepsilon}{r^{9}}\right. \\
& +\frac{184 M^{6} \varepsilon^{2}}{r^{10}}-\frac{1016 M^{7} \varepsilon^{2}}{r^{11}}+\frac{1908 M^{8} \varepsilon^{2}}{r^{12}} \\
& +\frac{160 M^{9} \varepsilon^{3}}{r^{13}}-\frac{804 M^{10} \varepsilon^{3}}{r^{14}}+\frac{1632 M^{11} \varepsilon^{3}}{r^{15}} \\
& +\frac{69 M^{12} \varepsilon^{4}}{r^{16}}-\frac{176 M^{13} \varepsilon^{4}}{r^{17}}+\frac{432 M^{14} \varepsilon^{4}}{r^{18}} \\
& \left.+\frac{16 M^{15} \varepsilon^{5}}{r^{19}}+\frac{16 M^{16} \varepsilon^{5}}{r^{20}}+\frac{4 M^{18} \varepsilon^{6}}{r^{22}}\right) .
\end{aligned}
$$
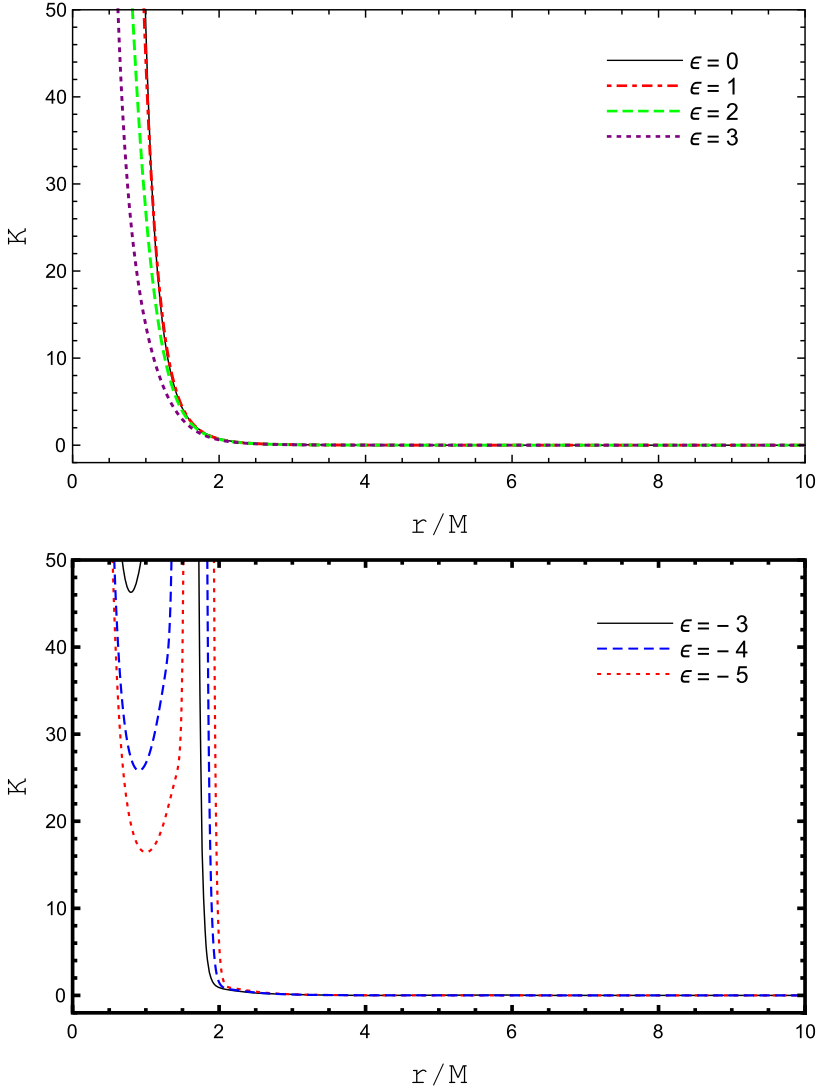

Fig. 1 Kretschmann scalar, given by Eq. (5), for some non-negative (top panel) and negative (bottom panel) values of the deformation parameter $\varepsilon$

This scalar plays an important role in BH physics, enabling us to determine the spacetime singularities. By analyzing Eq. (5) for positive values of $\varepsilon$, we note that the scalar $K$ diverges at $r=0$, as in Schwarzschild spacetime $(\varepsilon=0)$. When considering $\varepsilon<0$, the Kretschmann scalar diverges for two different radii: the usual $r=0$ and the additional radius $r=|\varepsilon|^{1 / 3} M$, which defines a surface-like singularity. In Fig. 1, we plot the Kretschmann scalar for positive (top panel) and negative (bottom panel) values of the deformation parameter $\varepsilon$.

If $\varepsilon$ is sufficiently negative, the surface-like singularity lies outside the $\mathrm{BH}$ event horizon, being located on the event horizon for $\varepsilon=-8$, as it can be seen in Fig. 2, and outside the event horizon for $\varepsilon<-8$. Therefore, if the deformation parameter is smaller than -8 , the line element (4) is associated to a naked singularity, and then violates the cosmic censorship conjecture [37]. Throughout this paper we consider $\varepsilon>-8$. 


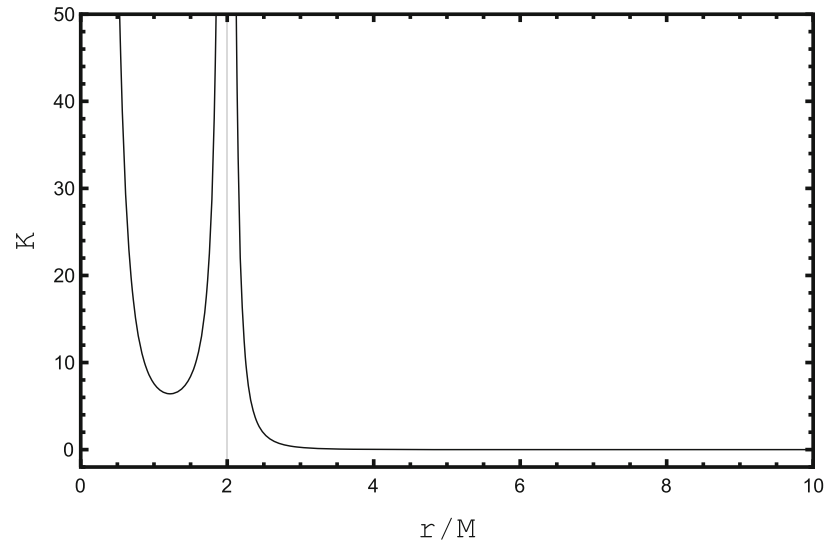

Fig. 2 Kretschmann scalar for $\varepsilon=-8$, with the surface-like singularity located on the event horizon of the JPBH

\section{Light-like trajectories}

We can obtain the trajectories of particles in a curved spacetime with covariant metric components $g_{\mu \nu}$, considering the Lagrangian

$L=\frac{1}{2} g_{\mu \nu} \dot{x}^{\mu} \dot{x}^{\nu}$,

where the overdot represents a derivative with respect to the affine parameter. The 4-velocity $\dot{x}^{\mu}$ is normalized to unity for massive particles $\left(\dot{x}^{\mu} \dot{x}_{\mu}=1\right)$ and has vanishing norm $\left(\dot{x}^{\mu} \dot{x}_{\mu}=0\right)$ for massless particles, so that for photons we have

$L=0$.

Since the line element (4) is spherically symmetric, without loss of generality we may evaluate Eq. (7) in the equatorial plane $(\theta=\pi / 2)$, and obtain the orbit equation for massless particles, given by

$$
\left(\frac{d u}{d \phi}\right)^{2}=-u^{2}\left(\frac{1-2 M u}{1+\varepsilon M^{3} u^{3}}\right)+\frac{1}{b^{2}\left(1+\varepsilon M^{3} u^{3}\right)^{2}},
$$

where $u(\phi) \equiv 1 / r(\phi)$ and $b$ is the impact parameter [2]. Equation (8) allows an unstable circular orbit (also called light ring or light sphere) at $r=r_{c}$. To determine the critical radius $r_{c}$ for different values of the deformation parameter $\varepsilon$, we impose that $d u / d \phi=0$, and $d^{2} u / d \phi^{2}=0$. We plot the light sphere radius $\left(r_{c}\right)$ for several JPBHs in Fig. 3.

There is a critical impact parameter $b=b_{c}$, so that any photon, with impact parameter equal to $b_{c}$, incoming from spatial infinity stays trapped in the light sphere at $r=r_{c}$. The expression for the critical impact parameter $b_{c}$ as a function of the JP parameter $\varepsilon$ is cumbersome and we prefer not to show it here. We plot the critical impact parameter for different JPBHs in Fig. 4.

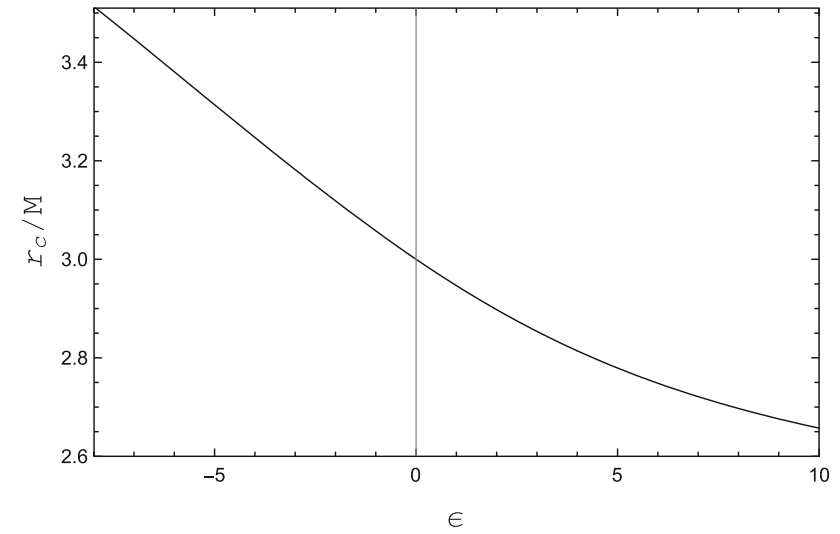

Fig. 3 Light sphere location $\left(r_{c}\right)$, plotted as a function of the JPBH deformation parameter $\varepsilon$

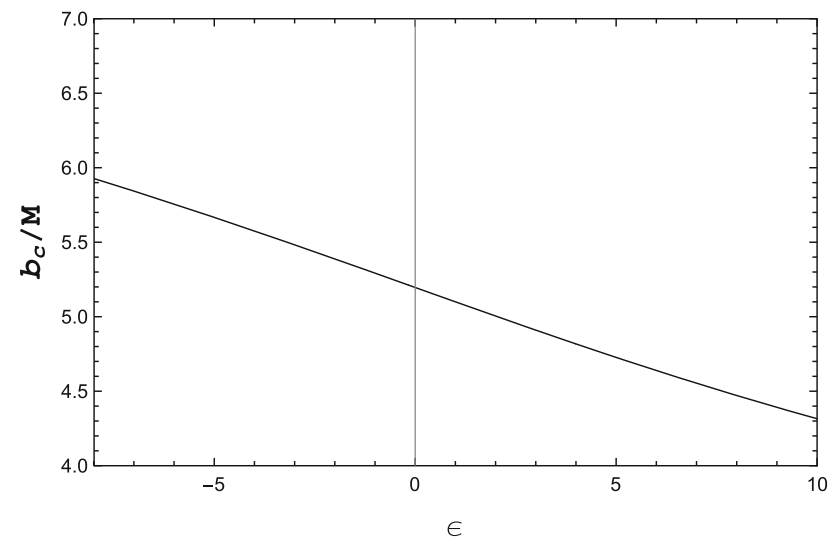

Fig. 4 Critical impact parameter $b_{c}$, plotted as a function of $\varepsilon$ for JPBHs

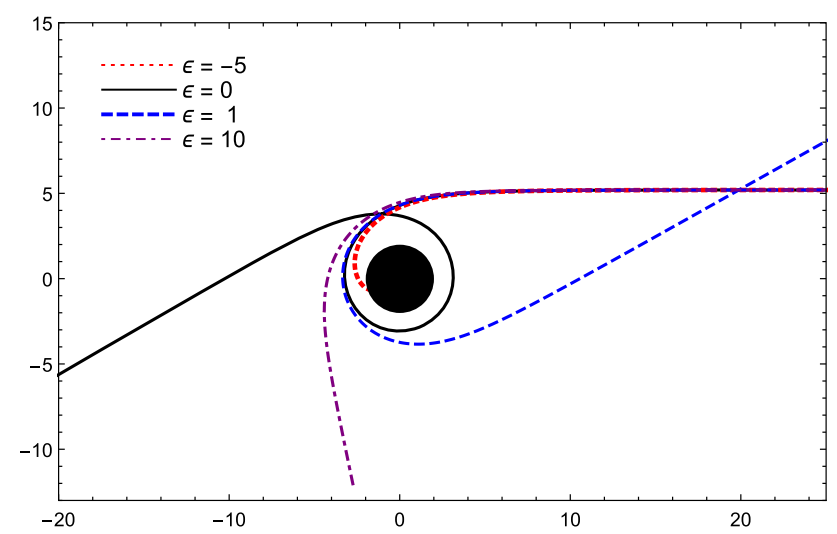

Fig. 5 Null geodesics with $b=5.2 M$, considering different choices of $\varepsilon$. We note that the JPBH has a stronger influence in photon trajectories for smaller values of the deformation parameter

In Fig. 5 we plot the photon trajectories for different choices of the JP parameter $\varepsilon$, fixing the impact parameter $b=5.2 \mathrm{M}$. We note that, light rays are less deflected by JPBHs with a bigger deformation parameter. 

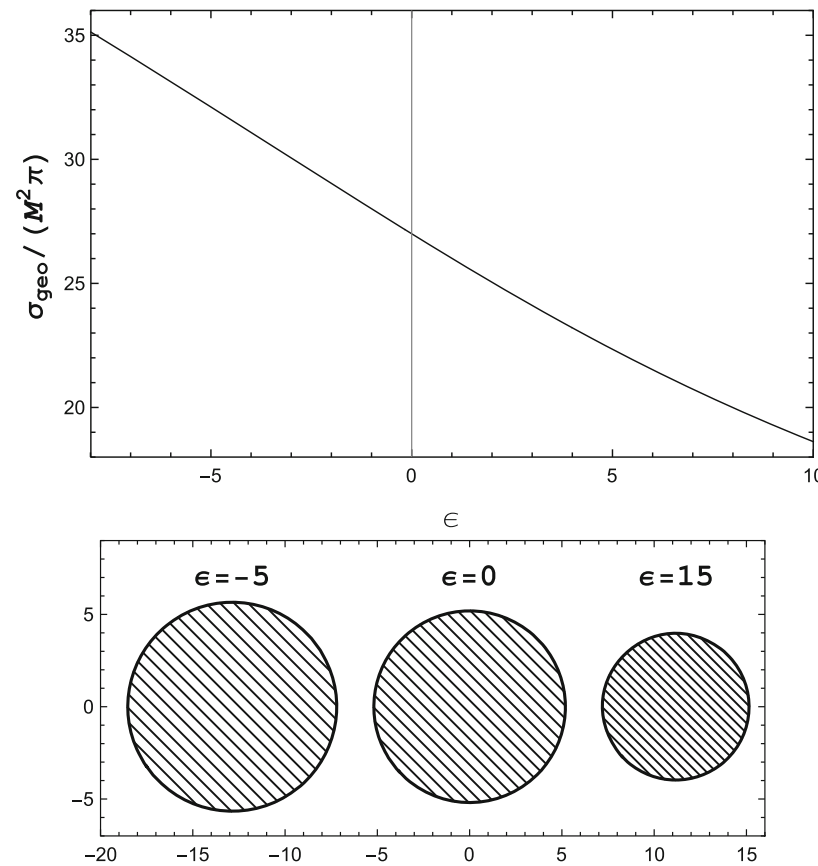

Fig. 6 Top: classical absorption cross section, as a function of $\varepsilon$. Bottom: geometrical representation of the classical absorption cross section for different choices of $\varepsilon$

The geometrical (or classical) absorption cross section is given by the area of a disk with radius $b_{c}$, namely

$\sigma_{g e o}=\pi b_{c}^{2}$.

From Fig. 6 we notice that as the deformation parameter of the JPBH increases, the geometrical absorption cross section diminishes.

In Sect. 5 we use this classical analysis as a consistency check of our numerical results in the high-frequency regime.

\section{Massless scalar field dynamics}

In order to study the absorption of massless scalar waves by Schwazschild-like BHs, we consider a spin-0 field governed by the (massless) Klein-Gordon equation,

$(-g)^{-1 / 2} \partial_{\mu}\left(g^{\mu \nu} \sqrt{-g} \partial_{\nu} \Psi\right)=0$,

with $\partial_{\mu} \equiv \partial / \partial x^{\mu}$ and $g^{\mu \nu}$ being the contravariant metric components. For the JPBH, the metric determinant $g$ is

$g=-\frac{\left(r^{3}+M^{3} \varepsilon\right)^{2}}{r^{2}} \sin ^{2} \theta$.

Due to the spherical symmetry, the massless scalar field $\Psi$ can be conveniently decomposed as follows

$\Psi\left(x^{\mu}\right)=\frac{\psi_{\omega l}(r)}{r} Y_{l m}(\theta, \phi) e^{-i \omega t}$,

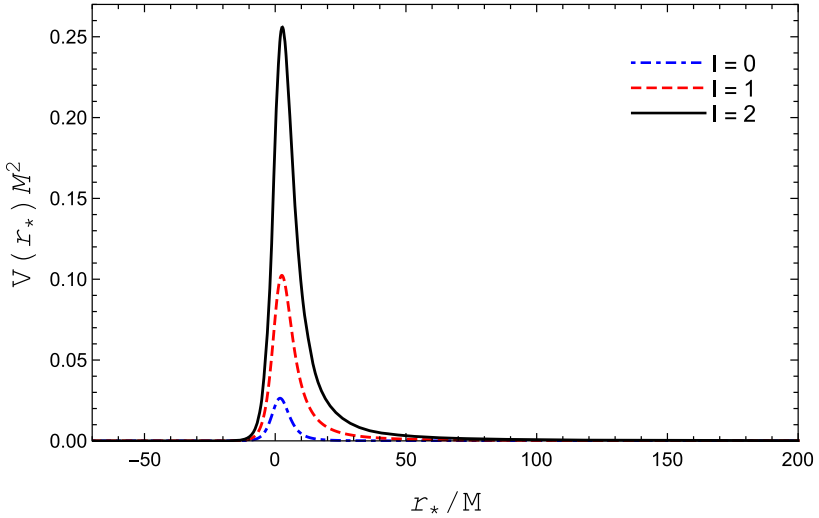

Fig. 7 Effective potential with $\varepsilon=1$, for modes $l=0$ (blue dotdashed line), $l=1$ (red dashed line) and $l=2$ (black solid line)

where $Y_{l m}(\theta, \phi)$ are the spherical harmonics with eigenvalues $l(l+1)$. By substituting Eq. (12) in Eq. (10) we obtain the ordinary differential equation for the radial function $\psi_{\omega l}(r)$, namely

$f(r) \frac{d}{d r}\left[f(r) \frac{d \psi_{\omega l}}{d r}\right]+\left[\omega^{2}-V_{l}(r)\right] \psi_{\omega l}=0$,

where $V_{l}$ is the effective potential, given by

$V_{l}(r) \equiv \frac{f(r)}{r} \frac{d f}{d r}+f(r) \frac{l(l+1)}{r^{2}}\left[1+\varepsilon\left(\frac{M}{r}\right)^{3}\right]$.

Compared with the well-known Schwarzschild case, the effective potential presents an extra $\varepsilon$ dependent term. We note that the only deformed contribution in Eq. (14) is multiplied by the eigenvalue $l(l+1)$ of the spherical harmonics, so that the lower mode contribution $(l=0)$ coincides with the Schwarzschild one for any JPBH.

By introducing the tortoise coordinate $r_{\star}$, namely

$r_{\star} \equiv \int \frac{d r}{f(r)}$,

we can rewrite Eq. (13) as a Schrödinger-like equation:

$\frac{d^{2} \psi_{\omega l}}{d r_{\star}^{2}}+\left[\omega^{2}-V_{l}\right] \psi_{\omega l}\left(r_{\star}\right)=0$.

We notice in Fig. 7 that for a fixed deformation parameter $\varepsilon$, the peak of the effective potential is bigger as we increase the mode number $l$. For a fixed mode number $l$, the peak of the effective potential increases as we increase the deformation parameter $\varepsilon$, as it can be seen in Fig. 8 .

In order to investigate the absorption of massless scalar waves, we seek for solutions of Eq. (16) that are purely incoming waves at the event horizon and a composition of ingoing and outgoing waves at spatial infinity, i.e, that satisfy the following boundary conditions:

$\psi_{\omega l}\left(r_{\star}\right) \sim \begin{cases}e^{-i \omega r_{\star}}+\mathscr{R}_{\omega l} e^{i \omega r_{\star}}, & r_{\star} \rightarrow+\infty, \\ \mathscr{T}_{\omega l} e^{-i \omega r_{\star}}, & r_{\star} \rightarrow-\infty .\end{cases}$ 

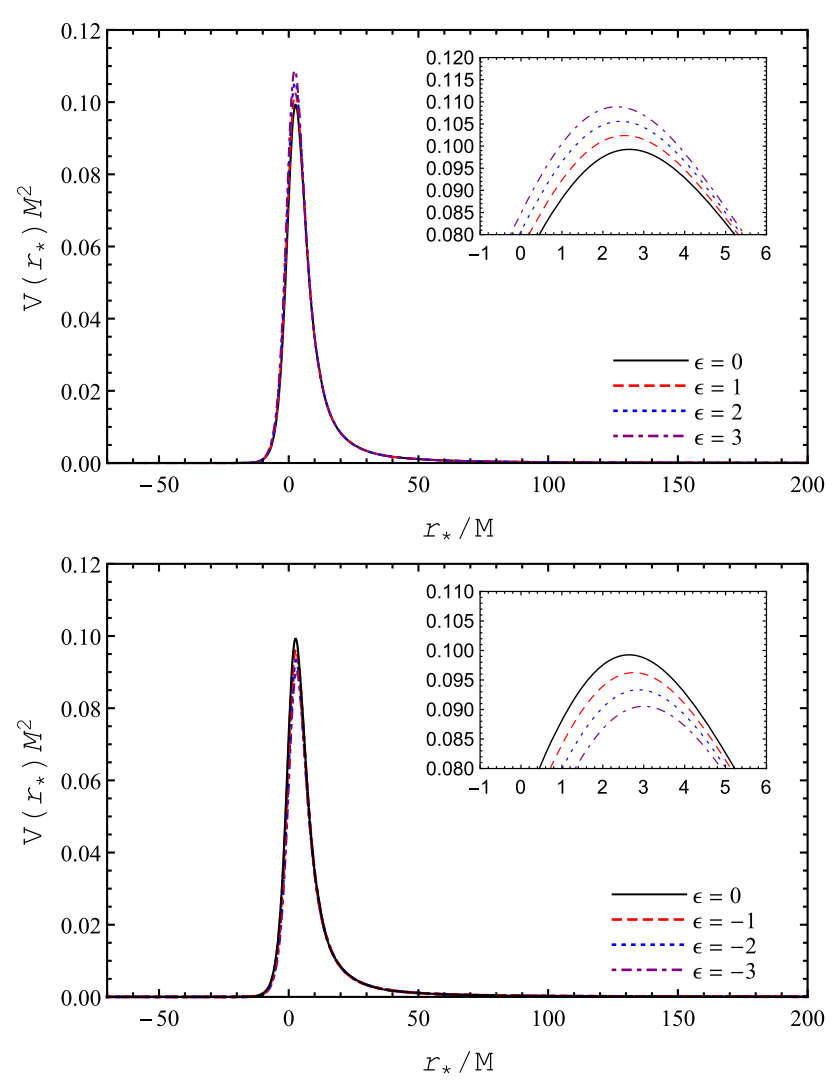

Fig. 8 Effective potential, given by Eq. (14), with $l=1$, for nonnegative (top panel) and non-positive (bottom panel) values of $\varepsilon$

The reflection and transmission coefficients are related to $\mathscr{R}_{\omega l}$ and $\mathscr{T}_{\omega l}$, respectively. Due to the flux conservation, the following relation is satisfied:

$\left|\mathscr{R}_{\omega l}\right|^{2}+\left|\mathscr{T}_{\omega l}\right|^{2}=1$.

\section{Absorption}

The absorption cross section can be defined as the ratio between the number of particles absorbed by the black hole and the incident particle flux. One can use the partial waves method to obtain the total absorption cross section of a scalar field [38], namely:

$\sigma=\sum_{l=0}^{\infty} \sigma_{l}$

where $\sigma_{l}$ are the partial absorption cross sections, defined as

$\sigma_{l} \equiv \frac{\pi}{\omega^{2}}(2 l+1) \Gamma_{l}(\omega)$.

The $\Gamma_{l}$ in Eq. (20) are the greybody factors, which are related to the absorption probability [39]. Considering the asymp- totic expansion (17), the greybody factors may be written as

$\Gamma_{l}=\left|\mathscr{T}_{\omega l}\right|^{2}$.

The total absorption cross section is well-known for static BHs in the low- and high-frequency regimes. In the lowfrequency regime Higuchi found that the massless scalar absorption cross section goes to the area of the event horizon for any stationary BH spacetime [40,41]. In the highfrequency regime the absorption cross section oscillates around the geometrical capture cross section (9), and we can use the analytical sinc approximation (cf. Sect. 5.1) to determine the high-frequency behaviour.

In the remaining of this section we compute the total absorption cross section using both the sinc approximation and the numerical method discussed in Ref. [42].

\subsection{Sinc approximation}

Sanchez proposed, to the Schwarzschild BH case, the following analytical approximation for the total scalar absorption cross section, in the high-frequency regime:

$\sigma_{\text {San }}=\frac{27 \pi}{4}-\frac{A}{\omega r_{h}} \sin \pi(3 \sqrt{3})\left(\omega r_{h}+B\right)$,

where $A=1.41 \sim \sqrt{2}$ and $B<10^{-4}$ give the best fit. This approximation has been obtained analytically in Ref. [43] for Schwarzschild BHs, in the high-frequency regime, based in an analytical extension of the greybody factor, and summing over $l$-modes using the Poisson sum formula. Following Ref. [43], one can write the approximation for the scalar absorption by a Schwarzschild $\mathrm{BH}$ as:

$\sigma_{a b s}=\sigma_{g e o}+\sigma_{R P}+\mathscr{O}\left(\frac{1}{\omega^{2}}\right)$,

where $\sigma_{g e o}$ is given by Eq. (9), and $\sigma_{R P}$ is a sum over Regge poles, given by

$\sigma_{R P}=-\frac{4 \pi^{2}}{\omega^{2}} \operatorname{Re}\left(\sum_{n=1}^{+\infty} \frac{\lambda_{n}(\omega) \gamma_{n}(\omega) e^{i \pi\left(\lambda_{n}(\omega)-1 / 2\right)}}{\sin \left[\pi\left(\lambda_{n}(\omega)-1 / 2\right)\right]}\right)$.

Here $\lambda_{n}$ are the Regge poles and $\gamma_{n}$ are the residues of the greybody factor.

Décanini et al. generalized the Sanchez approximation (22), for an arbitrary static and spherically symmetric BH, obtaining that the total scalar absorption cross section in the high frequency limit, in a four dimensional spacetime, is [43]

$\sigma_{a b s}^{h f}=\sigma_{g e o}\left[1-8 \pi \beta e^{-\pi \beta} \operatorname{sinc}\left(\frac{2 \pi \omega}{\Omega_{0}}\right)\right]$,

where the sine cardinal is defined as $\operatorname{sinc}(z) \equiv \sin (z) / z$. The $\Omega_{0}$ is the orbital frequency, which is the inverse of the critical 

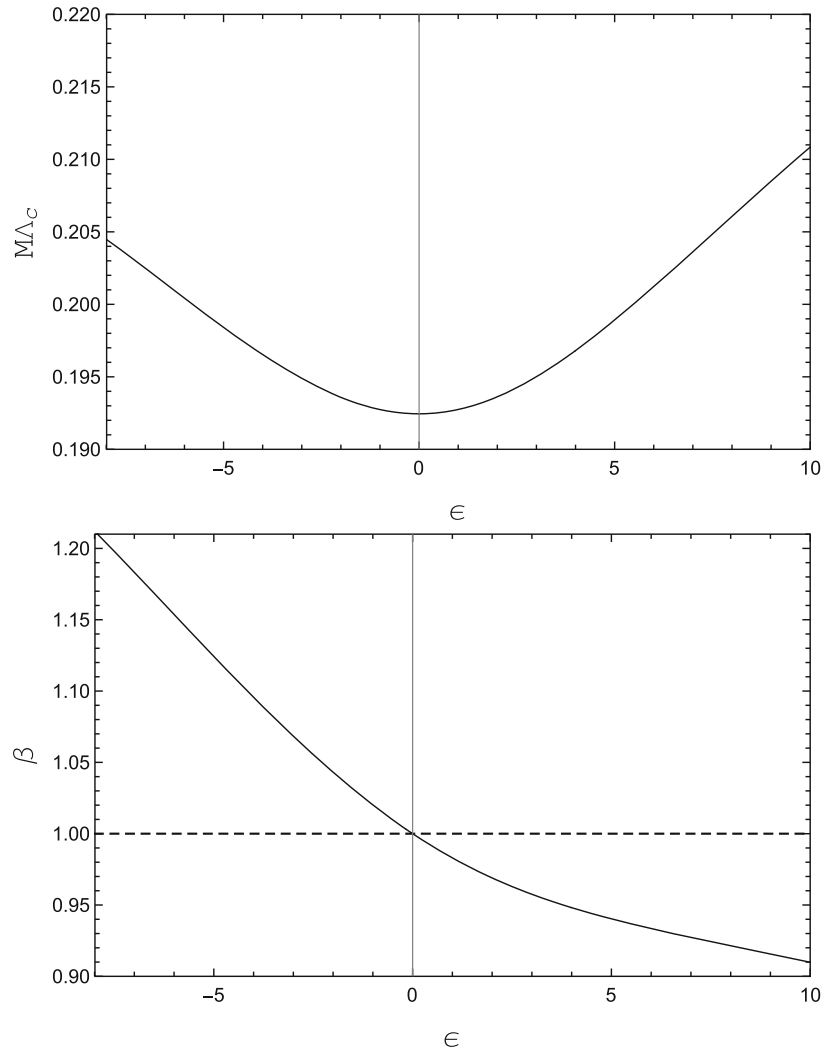

Fig. 9 Top: Lyapunov exponent $\Lambda_{c}$ at the unstable circular orbit radius $r_{c}$, as a function of $\varepsilon$. Bottom: $\beta$ factor as a function of $\varepsilon$. When the deformation parameter vanishes the $\beta$ factor is equal to 1

impact parameter $b_{c}$ [44] plotted in Fig. 4. The $\beta$ factor is related to the Lyapunov exponent $\Lambda$, by [45]

$\beta=\frac{\Lambda_{c}}{\Omega_{0}}$,

where $\Lambda_{c}$ is the Lyapunov exponent at the unstable circular orbits radius $r_{c}$, as introduced in Ref. [44]. The Lyapunov exponent $\Lambda_{c}$ and the $\beta$ factor are plotted in Fig. 9. Their expressions are cumbersome for JPBH, and we prefer not to show them here. If the deformation parameter vanishes, $\beta=1$ (cf. bottom panel of Fig. 9), and we recover the for Schwarzschild case.

\subsection{Numerical method}

In order to obtain the total absorption cross section in the whole frequency range, we solve the radial equation (16) numerically, imposing the boundary conditions (17). The integration is made from close enough to the event horizon to a sufficiently large value of the radial coordinate. We compare the radial solution obtained numerically with the asymptotic solution (17), finding the reflection and transmission coefficients. For more details of the numerical method and the convergence of the solution, see Ref. [42].

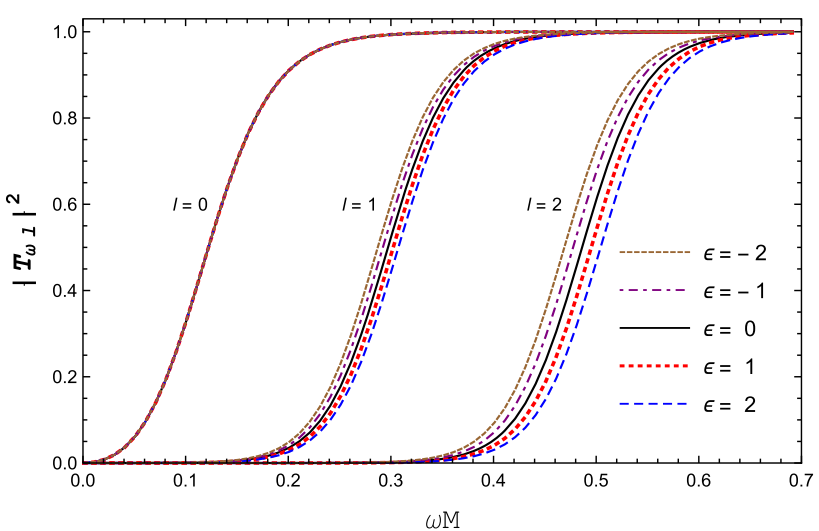

Fig. 10 Transmission coefficients for the first three modes $(l=0$, $l=1$ and $l=2$ ), for different choices of the deformation parameter $(\varepsilon=-2,-1,0,1$ and 2$)$. The transmission coefficients for $l=0$ are the same for any value of the deformation parameter, while for non-vanishing angular momentum they are different, and the difference increases for higher values of $l$

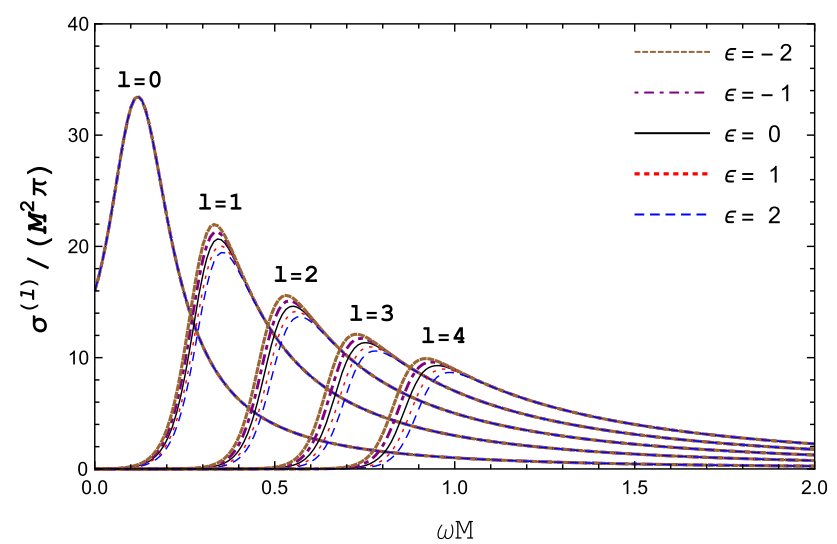

Fig. 11 Partial absorption cross sections for different choices of the deformation parameter $\varepsilon$

Applying this numerical method we can obtain the transmission coefficients for different values of the deformation parameter, which we plot in Fig. 10. We calculate the partial absorption cross sections for different values of $\varepsilon$, by plugging the numerical transmission coefficients into Eq. (20), and plot them in Fig. 11. In Figs. 12 and 13 we exhibit the total absorption cross sections, obtained by summing over the $l$ modes [cf. Eq. (19)], and compare them with the wellknown Schwarzschild case $(\varepsilon=0)$. We see that as the deformation parameter increases, the total absorption diminishes. We also notice, both in Fig. 12 and in Fig. 13 that, for different values of $\varepsilon$, the first peak remains almost unaltered, while the other peaks change significantly.

As it can be seen in Fig. 14, our numerical results agree very well with the sinc approximation in the high-frequency regime.

For the Schwarzschild case, as pointed out by Unruh in Ref. [26], the zero-frequency limit is given by the contribu- 


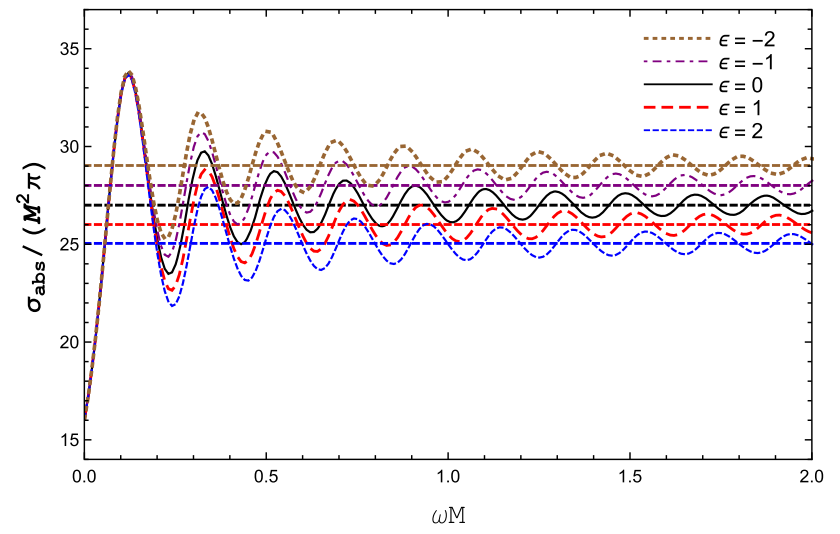

Fig. 12 Total absorption cross section of Schwarzschild-like objects for different values of $\varepsilon$. The horizontal dashed lines represent the geometrical absorption cross sections in each case

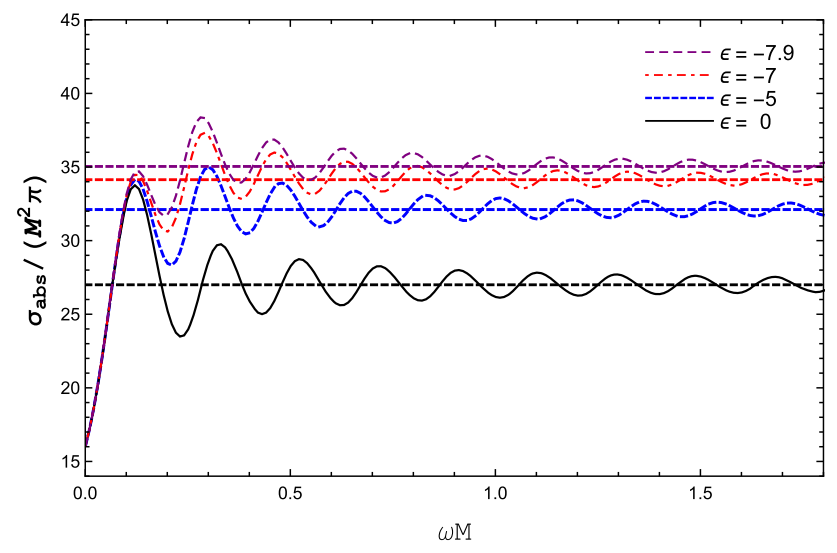

Fig. 13 Total absorption cross section of JPBHs for some non-positive values of $\varepsilon$. Although the first peak $(l=0)$ does not change much, the second peak $(l=1)$ increases significantly as we decrease the deformation parameter $\varepsilon$

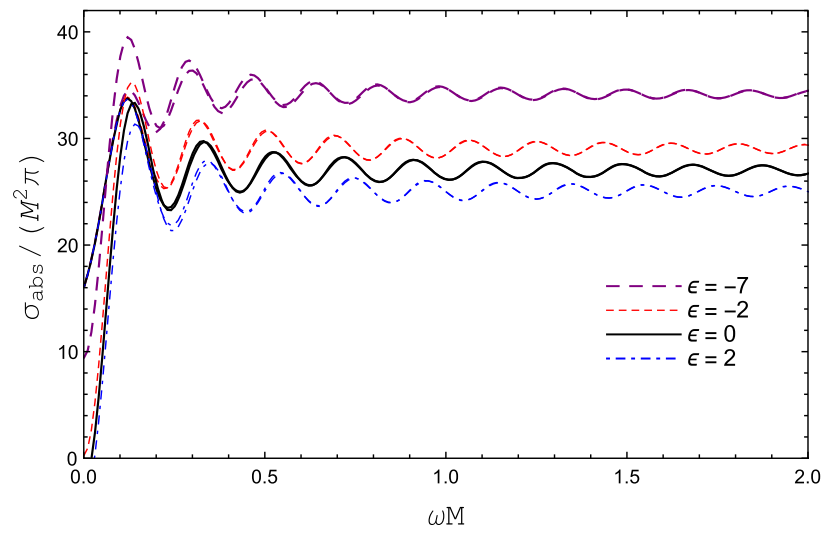

Fig. 14 Comparison of the sinc approximation with the numerical solution for four values of the deformation parameter $(\varepsilon=-7, \varepsilon=-2$, $\varepsilon=0$, and $\varepsilon=2$ )

tion of the lowest mode $l=0$, being equal to the area of the event horizon. We notice that the same happens to the JPBH, independently of the non-vanishing deformation parameter.
This can also be understood from the behavior of the transmission coefficients of the JPBH (cf. Fig. 10), by noticing that, in the low-frequency regime, the transmission coefficient for $l=0$ is dominant. Due the dependence of the effective potential (14) on the deformation parameter, the radial solution for the lowest mode $l=0$ is the same, independently of the value of $\varepsilon$. Hence, the transmission coefficient for $l=0$ is the same for any JPBH. Therefore, in the low-frequency regime the total absorption cross section goes to the area of the JPBH, what is consistent with the general result for the scalar absorption of static BHs [46].

\section{Final remarks}

Over the years, several propositions to include new parameters on BH solutions, violating the no-hair theorems, have been presented. In 2011, Johannsen and Psaltis, without requiring the Einstein's equations to be satisfied, proposed a Schwarzschild-like spacetime, regular on and outside the event horizon. The JPBH has an additional parameter, so that two JPBHs with the same mass can deform the spacetime differently.

We have investigated the scalar absorption by JPBHs. We have derived the orbit equation for light rays around JPBHs and solved it to obtain the critical impact parameter and the geometrical absorption cross section, as functions of the deformation parameter. We have shown that if the value of the deformation parameter is increased, both critical impact parameter and geometrical absorption cross section diminishes.

We have used numerical techniques to compute the partial and total absorption cross sections. We obtained that, as the deformation parameter is increased, the absorption of the scalar field by the JPBH decreases. We also obtained that in the high-frequency regime the total absorption cross section oscillates around the geometrical absorption cross section. In the low-frequency regime, the absorption cross section goes to the area of the black hole, which is independent of the deformation parameter value.

As a consistency check of our numerical results, we used the sinc approximation to compute the total absorption cross section in the high-frequency regime, obtaining excellent concordance.

Acknowledgements The authors would like to acknowledge Conselho Nacional de Desenvolvimento Científico e Tecnológico (CNPq) and Coordenação de Aperfeiçoamento de Pessoal de Nível Superior (CAPES) - Finance Code 001, from Brazil, for partial financial support. This research has also received funding from the European Union's Horizon 2020 research and innovation programme under the H2020MSCA-RISE-2017 Grant no. FunFiCO-777740. The authors are grateful to Atsushi Higuchi for profitable discussions. 
Data Availability Statement This manuscript has no associated data or the data will not be deposited. [Authors' comment: This is a theoretical study and has no experimental data associated to it.]

Open Access This article is licensed under a Creative Commons Attribution 4.0 International License, which permits use, sharing, adaptation, distribution and reproduction in any medium or format, as long as you give appropriate credit to the original author(s) and the source, provide a link to the Creative Commons licence, and indicate if changes were made. The images or other third party material in this article are included in the article's Creative Commons licence, unless indicated otherwise in a credit line to the material. If material is not included in the article's Creative Commons licence and your intended use is not permitted by statutory regulation or exceeds the permitted use, you will need to obtain permission directly from the copyright holder. To view a copy of this licence, visit http://creativecomm ons.org/licenses/by/4.0/.

Funded by SCOAP ${ }^{3}$.

\section{References}

1. P.T. Chrusciel, J.L. Costa, M. Heusler, Stationary black holes: uniqueness and beyond. Living Rev. Relat. 15, 7 (2012)

2. R.M. Wald, General relativity (University of Chicago Press, Chicago, 2010)

3. E. Berti, V. Cardoso, C.M. Will, Gravitational-wave spectroscopy of massive black holes with the space interferometer LISA. Phys. Rev. D 73, 064030 (2006)

4. E. Berti, A. Sesana, E. Barausse, V. Cardoso, K. Belczynski, Spectroscopy of Kerr Black Holes with Earth- and Space-Based Interferometers. Phys. Rev. Lett. 117, 101102 (2016)

5. E. Berti et al., Testing general relativity with present and future astrophysical observations. Class. Quantum Gravity 32, 243001 (2015)

6. S. Gossan, J. Veitch, B.S. Sathyaprakash, Bayesian model selection for testing the no-hair theorem with black hole ringdowns. Phys. Rev. D 85, 124056 (2012)

7. J. Meidam, M. Agathos, C. Van Den Broeck, J. Veitch, B.S. Sathyaprakash, Testing the no-hair theorem with black hole ringdowns using TIGER. Phys. Rev. D 90, 064009 (2014)

8. M. Isi, M. Giesler, W.M. Farr, M.A. Scheel, S.A. Teukolsky, Testing the no-hair theorem with GW150914. Phys. Rev. Lett. 123, 111102 (2019)

9. K. Akiyama et al. (Event Horizon Telescope Collaboration), First M87 event horizon telescope results. VI. The shadow and mass of the central black hole. Astrophys. J. 875, L6 (2019)

10. K. Akiyama et al. (Event Horizon Telescope Collaboration), First M87 event horizon telescope results. II. Array and instrumentation 875, L2 (2019)

11. K. Akiyama et al. (Event Horizon Telescope Collaboration), First M87 event horizon telescope results. III. Data processing and calibration 875, L3 (2019)

12. K. Akiyama et al. (Event Horizon Telescope Collaboration), First M87 event horizon telescope results. IV. Imaging the central supermassive black hole, 875, L4 (2019)

13. K. Akiyama et al. (Event Horizon Telescope Collaboration), First M87 event horizon telescope results. V. Physical origin of the asymmetric ring, 875, L5 (2019)

14. B.P. Abbott et al. (LIGO Scientific and Virgo Collaborations), GW151226: observation of gravitational waves from a 22-solarmass binary black hole coalescence. Phys. Rev. Lett. 116, 241103 (2016)
15. B.P. Abbott et al. (LIGO Scientific and Virgo Collaborations), Observation of gravitational waves from a binary black hole merger 116, 061102 (2016)

16. N.A. Collins, S.A. Hughes, Towards a formalism for mapping the spacetimes of massive compact objects: bumpy black holes and their orbits. Phys. Rev. D 69, 124022 (2004)

17. S.J. Vigeland, S.A. Hughes, Spacetime and orbits of bumpy black holes. Phys. Rev. D 81, 024030 (2010)

18. S. Vigeland, N. Yunes, L. Stein, Bumpy black holes in alternative theories of gravity. Phys. Rev. D 83, 104027 (2011)

19. T. Johannsen, D. Psaltis, Metric for rapidly spinning black holes suitable for strong-field tests of the no-hair theorem. Phys. Rev. D 83, 124015 (2011)

20. R. Rahim, K. Saifullah, Charging the Johannsen-Psaltis spacetime. Ann. Phys. (N. Y.) 405, 220 (2019)

21. K. Glampedakis, G. Pappas, Modification of photon trapping orbits as a diagnostic of non-Kerr spacetimes. Phys. Rev. D 99, 124041 (2019)

22. V. Cardoso, P. Pani, J. Rico, On generic parametrizations of spinning black-hole geometries. Phys. Rev. D 89, 064007 (2014)

23. L. Rezzolla, A. Zhidenko, New parametrization for spherically symmetric black holes in metric theories of gravity. Phys. Rev. D 90, 084009 (2014)

24. R. Konoplya, A. Zhidenko, Detection of gravitational waves from black holes: Is there a window for alternative theories? Phys. Lett. B 756, 350 (2016)

25. R. Fabbri, Scattering and absorption of electromagnetic waves by a Schwarzschild black hole. Phys. Rev. D 12, 933 (1975)

26. W.G. Unruh, Absorption cross section of small black holes. Phys. Rev. D 14, 3251 (1976)

27. N.G. Sanchez, Absorption and emission spectra of a Schwarzschild black hole. Phys. Rev. D 18, 1030 (1978)

28. C.L. Benone, E.S. Oliveira, S.R. Dolan, L.C.B. Crispino, Absorption of a massive scalar field by a charged black hole. Phys. Rev. D 89, 104053 (2014)

29. C.F.B. Macedo, L.C.B. Crispino, Absorption of planar massless scalar waves by Bardeen regular black holes. Phys. Rev. D 90, $064001(2014)$

30. L.C.B. Crispino, S.R. Dolan, A. Higuchi, E.S. Oliveira, Scattering from charged black holes and supergravity. Phys. Rev. D 92, 084056 (2015)

31. C.L. Benone, L.C.B. Crispino, Superradiance in static black hole spacetimes. Phys. Rev. D 93, 024028 (2016)

32. L.C.S. Leite, S.R. Dolan, L.C.B. Crispino, Absorption of electromagnetic and gravitational waves by Kerr black holes. Phys. Lett. B 774, 130 (2017)

33. L.C.S. Leite, S.R. Dolan, L.C.B. Crispino, Absorption of electromagnetic plane waves by rotating black holes. Phys. Rev. D 98, 024046 (2018)

34. L.C.S. Leite, C.L. Benone, L.C.B. Crispino, On-axis scattering of scalar fields by charged rotating black holes. Phys. Lett. B 795, 496 (2019)

35. J.G. Williams, S.G. Turyshev, D.H. Broggs, Progress in Lunar Laser Ranging Tests of Relativistic Gravity. Phys. Rev. Lett. 93, 261101 (2004)

36. B. Bertotti, L. Iess, P. Tortora, A test of general relativity using radio links with the Cassini spacecraft. Nature (London) 425, 374 (2003)

37. R. Penrose, Gravitational collapse: the role of general relativity. Riv Nouvo Cim 1, 252 (1969)

38. L.C.B. Crispino, S.R. Dolan, E.S. Oliveira, Scattering of massless scalar waves by Reissner-Nordström black holes. Phys. Rev. D 79, 064022 (2009)

39. L.C.B. Crispino, A. Higuchi, E.S. Oliveira, J.V. Rocha, Greybody factors for nonminimally coupled scalar fields in Schwarzschild-de Sitter spacetime. Phys. Rev. D 87, 104034 (2013) 
40. A. Higuchi, Low-frequency scalar absorption cross sections for stationary black holes. Class. Quantum Gravity 18, L139 (2001)

41. A. Higuchi, Addendum to 'Low-frequency scalar absorption cross sections for stationary black holes. Class. Quantum Gravity 19, 599(A) (2002)

42. S.R. Dolan, E.S. Oliveira, L.C.B. Crispino, Scattering of sound waves by a canonical acoustic hole. Phys. Rev. D 79, 064014 (2009)

43. Y. Décanini, G. Esposito-Farèse, A. Folacci, Universality of highenergy absorption cross sections for black holes. Phys. Rev. D 83, $044032(2011)$

44. V. Cardoso, A.S. Miranda, E. Berti, H. Witek, V.T. Zanchin, Geodesic stability, Lyapunov exponents, and quasinormal modes. Phys. Rev. D 79, 064016 (2009)
45. Y. Décanini, A. Folacci, B. Raffaelli, Unstable circular null geodesics of static spherically symmetric black holes, Regge poles, and quasinormal frequencies. Phys. Rev. D 81, 104039 (2010)

46. S.R. Das, G. Gibbons, S.D. Mathur, Universality of low energy absorption cross sections for black holes. Phys. Rev. Lett. 78, 417 (1997) 